\title{
Incorporación del acceso solar en la planificación urbana de las ciudades chilenas
}

Luis Merino. Universidad de Concepción, Concepción, Chile.

Ángel Hernández. Universidad de Concepción, Concepción, Chile. Thibaut Vermeulen. Universidad de Concepción, Concepción, Chile. Claudia García. Universidad de Concepción, Concepción, Chile.

RESUMEN | La radiación solar en edificaciones es clave para asegurar su confort térmico y lumínico. El acceso a ella se asegura a través de restricciones urbanísticas de edificación, que buscan asegurar un cierto nivel de exposición al sol en términos temporales (horas), energéticos (Joule) o una mezcla de ellos. El objetivo de este trabajo consiste en presentar una herramienta de planificación urbana denominada Líneas de Sección, la cual permite definir la altura máxima de las edificaciones del tejido urbano en las que se aplica, de tal manera que se asegure un mínimo de horas de exposición al sol en las fachadas norte, este y oeste de cada edificación durante algún periodo del año. En comparación con el criterio actual de resguardo solar en Chile, la rasante, la aplicación de las Líneas de Sección permite un ingreso hasta diez veces mayor de energía solar en las fachadas norte durante el periodo invernal.

PALABRAS CLAVE | planificación urbana, medioambiente urbano, desarrollo sustentable.

ABSTRACT | Solar radiation in the urban built environment is a key aspect to assure luminous and thermal comfort. Solar access is regulated by urban restrictions for buildings, which seek to ensure a certain level of exposure to the sun in terms of time (hours), energy (Joule), or a mixture of them. The main goal of this work is to present an urban planning tool called Section Lines, which allows to define the maximum height of the buildings, in such a way that a minimum of sun exposure hours are assured on the north, east and west facades of the buildings during some period of time during the year. Compared to the current standard of solar access in Chile "rasante", the application of Section Lines allows to increase up to ten times the solar radiation on the north facades during the winter season.

KEYWORDS | urban planning, urban environment, sustainable development. 


\section{Introducción}

En el año 2018, cerca del 55\% de la población mundial habitaba en áreas urbanas, y se proyecta que esta cifra aumente a un 68\% para el año 2050 (Organización de las Naciones Unidas [onu], 2018). En Chile, las cifras del Censo 2017 arrojaron que el $87,8 \%$ de los habitantes vive en zonas urbanas (Instituto Nacional de Estadísticas [INE], 2018). Debido a que el mundo continúa urbanizándose, lograr urbanizaciones sustentables es clave para un desarrollo exitoso de nuestra sociedad, lo que debe lograrse mediante políticas de planificación del crecimiento urbano que garanticen la minimización de externalidades negativas para la sociedad (onU, 2018). Esta planificación debe incorporar las distintas dimensiones de la sustentabilidad, por lo que requiere estar alineada con las políticas que impulsa el gobierno chileno en materia de eficiencia energética para el sector de edificación.

La Política Energética de Chile 2050 (Ministerio de Energía, 2015) establece metas como la incorporación de estándares OCDE de construcción eficiente a las edificaciones de uso público y edificaciones de uso residencial. En esta misma línea, la Estrategia Nacional de Construcción Sustentable desarrollada por el Ministerio de Vivienda y Urbanismo (MINVu, 2018) busca establecer estándares y buenas prácticas de diseño, construcción y operación de las viviendas, con el fin de mejorar el desempeńo ambiental mediante la incorporación de criterios de sustentabilidad.

En el sector residencial, la eficiencia en el uso de la energía depende principalmente de cuatro factores: diseño del edificio, eficiencia en los sistemas de calefacción, comportamiento del usuario y la geometría urbana (Baker \& Steemers, 2000). Esta última impacta sobre el acceso solar de una edificación, influyendo en la disponibilidad de energía y luz en las fachadas de los edificios.

McCann (2008) define el acceso solar como la capacidad de un bien inmueble de recibir los rayos de luz solar directa de manera continua, sin obstrucciones de otra propiedad (vegetación, edificios, etc.). Estos rayos de luz solar pueden ser aprovechados por sistemas activos de transformación de energía solar, tal como colectores solares fotovoltaicos, o para agua caliente sanitaria, que generalmente se instalan en la techumbre o fachadas de las edificaciones. Por otro lado, la fenestración de los edificios o las aberturas de su envolvente desempeñan un rol fundamental en el confort térmico y lumínico del inmueble (Sadineni, Madala \& Boehm, 2011). En periodos invernales, la fenestración puede ayudar a reducir el requerimiento energético para calefaccionar e iluminar el espacio interior de una edificación (Ratti, Baker \& Steemers, 2005). En periodos estivales, los rayos de sol que ingresan por las aberturas de la envolvente pueden producir sobrecalentamiento y molestia visual por deslumbramiento (Capeluto \& Ochoa, 2017), por lo que se usan sistemas de control solar fijos o móviles en el exterior del edificio, tales como aleros, lamas, persianas, vegetación, etc. (Lechner, 2015).

Para lograr un acceso solar adecuado, se necesitan regulaciones que establezcan zonas con una densidad de edificación adecuada a su sistema de actividades, definan alturas máximas de construcciones, distanciamientos mínimos entre edificios de una misma agrupación, restricción de vegetación u otro objeto que bloquee los rayos directos del sol, compensaciones a propietarios que se vean afectados por 
sombreamientos, entre otros. Una completa revisión de estas y otras medidas implementadas en distintas ciudades del hemisferio norte se pueden encontrar en McCann (2008). A partir de lo anterior, se desprende que conseguir un acceso solar no es una tarea sencilla, ya que tal condición es el resultado de una interacción simultánea de múltiples variables para un instante dado. Algunas de estas variables son el lugar de emplazamiento en el planeta dado por la latitud geográfica, la forma y orientación del tejido urbano, etc.. Esto deviene en algo más complejo si, además, se quiere asegurar un mínimo estándar cuantificable, tal como una cierta cantidad de horas de exposición directa al sol, o garantizar la incidencia de una energía solar mínima en alguna fachada.

Debido a la complejidad de análisis de todas estas variables, se requieren métodos simples que permitan al diseńador y proyectista aplicar regulaciones que aseguren niveles adecuados de asoleamiento (Capeluto, Yezioro, Bleiberg \& Shaviv, 2006). En esa línea, este artículo tiene por objetivo evaluar el impacto que tendría incorporar el acceso solar en proyectos de edificación en Chile, para lo cual comienza por presentar la herramienta de resguardo solar que se aplica en el país, llamada "rasante", así como sus limitaciones. Luego se describen métodos de aseguramiento de acceso solar usados en algunos países, poniendo énfasis en describir detalladamente la herramienta de aseguramiento de horas de exposición directa al sol llamada Líneas de Sección. Los múltiples casos que se pueden presentar al momento de aplicar la rasante y las Líneas de Sección obligan a fijar una grilla típica de ciudad y emplazamientos fijos, que se presentan en la sección de metodología. En la sección de resultados, se presentan las alturas máximas generadas al aplicar las Líneas de Sección en distintas ciudades. Esto se complementa con un análisis de sensibilidad de esta herramienta con respecto a las horas de aseguramiento de asoleamiento. Finalmente, se compara la morfología de edificación que genera la Línea de Sección con la obtenida mediante la rasante, usando como caso de estudio la ciudad de Concepción, así como las diferencias de energía solar que reciben los volúmenes en estos dos escenarios de densificación.

\section{El acceso solar en el diseño urbano}

Uno de los primeros conceptos sobre el aseguramiento solar en el diseńo urbano es el de "envolvente solar" (Knowles, 1981), noción que remite a una superficie que regula el desarrollo urbano dentro de límites imaginarios derivados del movimiento del sol y tiene como objeto establecer las alturas máximas de los edificios en etapas previas a la construcción para que no obstruyan el acceso solar de las edificaciones existentes (Knowles, 2003). Capeluto y Shaviv (1999, 2001) sugirieron la creación de dos conceptos diferentes de envolventes solares: la "envolvente de los derechos solares" y la "envolvente para los colectores solares". La primera establece una superficie que regula el diseño de las alturas máximas de los edificios para que estos no priven de acceso solar a aquellos existentes en el vecindario durante un periodo determinado del ańo. La envolvente para los colectores solares presenta la posición más baja posible de ventanas y/o colectores solares pasivos en la fachada de un edificio, de modo que reciban sol en el invierno y sombra en el verano. El volumen 
generado entre ambas envolventes se denomina "volumen solar", el cual es el espacio máximo que pueden ocupar los edificios en el diseño (Capeluto \& Shaviv, 2001).

Permitir el acceso solar es una tarea de los planificadores urbanos, los cuales entregan las regulaciones y disposiciones de las características geométricas de los edificios. Knowles (2003) afirma que los diseńadores no se preocupan por el costo de calefacción e iluminación a largo plazo. En términos simples, están a favor del crecimiento sobre el mantenimiento, ignorando las condiciones favorables de disponibilidad solar.

En Chile, el desarrollo inmobiliario del último tiempo ha provocado que la construcción en altura vaya aumentando e invadiendo la morfología urbana (Daher, 2013), lo que ha generado áreas de sombreamiento que afectan no solo el valor de los predios vecinos, sino también el confort térmico y lumínico de las edificaciones afectadas (Hachem, Athienitis \& Fazio, 2014; Ratti et al., 2005). Por lo tanto, construir morfologías urbanas con las consideraciones adecuadas de acceso solar mejoraría considerablemente el confort térmico, los requerimientos energéticos de las edificaciones y la oportunidad de captar la energía solar para diferentes usos en ciudades, tal como colectores solares (Okeil, 2010).

\section{Situación chilena: rasante}

En Chile, los Planes Reguladores Comunales, en conjunto con la Ordenanza General de Urbanismo y Construcciones (ogUC), establecen normas urbanísticas, tales como los distanciamientos a los deslindes, rasantes y sombras, entre otras, pero que en su conjunto no aseguran un resguardo solar adecuado para los edificios (Saldías, 2016). La norma asociada al aseguramiento solar es la rasante, la cual es una recta inclinada imaginaria que define la envolvente teórica dentro de la cual puede desarrollarse un proyecto de edificación (minvu, 2018). Las rasantes se levantan en todos los puntos que forman los deslindes con otros predios, y en el punto medio entre líneas oficiales del espacio público que enfrenta el predio, salvo que el predio colinde con un área verde pública, en cuyo caso las rasantes se aplicarán en el deslinde entre ambos (MINvu, 2018). La rasante se origina desde el nivel del suelo, u otro punto estipulado en el Plan Regulador, y se proyecta hacia el interior del predio con un ángulo de inclinación que depende del lugar de emplazamiento del proyecto de construcción. El objetivo de esto es limitar la altura de la edificación que se pretende construir en un predio.

En el artículo 2.6.3 de la OGUC se presentan tres valores para el ángulo de la rasante que deben respetar los proyectos de edificación según su emplazamiento geográfico: zona norte, 80 grados sexagesimales; zona centro, 70 grados; y zona sur, 60 grados. La diferencia entre estos tres casos obedece al intento de incorporar el ángulo de elevación del sol según la latitud geográfica, ya que mientras nos alejamos de la línea del ecuador, disminuye la altura angular del sol medida con respecto al plano horizontal. Si bien esta altura del sol varía a lo largo de un día dado y según el día del año, para una misma fecha tendrá una altura solar diferente según la latitud geográfica donde se encuentre el observador. La Figura 3 ilustra esta situación para distintas ciudades de Chile, donde podemos ver que, en el solsticio de invierno, 
al mediodía solar el sol presenta un ángulo de elevación de 12 grados y 47 grados sexagesimales en las ciudades de Punta Arenas e Iquique, respectivamente.

Los tres ángulos de rasante definidos en la OGUC generan un plano imaginario que limita las alturas de edificación. Ello provocó que proyectistas maximizaran el volumen construible de los edificios al generar superficies inclinadas paralelas y coincidentes con el plano imaginario de la rasante en los pisos superiores. Este tipo de morfologías del tipo piramidal de edificaciones se puede encontrar en distintas ciudades de Chile. Para evitar que los edificios tengan geometrías irregulares e inclinadas debido al criterio de la rasante, se incorporó el artículo 2.6.11 en la OGUC, que corresponde a la realización de un estudio comparativo de la sombra proyectada por la edificación, la cual da la libertad de sobrepasar el límite de altura con fachadas verticales, pero sin sobrepasar el volumen máximo generado por los planos de rasantes.

El criterio de la rasante es ambiguo, ya que la oguc no establece si se asegura un número de horas de sol durante un periodo específico del año (Merino, Vermeulen, Medina, Nahön \& Agurto, 2015). Saldías (2016) evaluó el criterio de la rasante propuesto por la OGUC usando cańones urbanos, y concluyó que no hay un resguardo solar para los periodos de otoño e invierno. Esto es válido para cualquier orientación y para las tres zonas establecidas en la oguc. Además, propuso estudiar el artículo 2.6.11 de la OGUC sobre las sombras generadas por los edificios, ya que en ningún caso corresponde a un resguardo solar.

\section{Métodos de aplicación para el aseguramiento solar}

Capeluto et al. (2006) establecen que los métodos para asegurar el acceso a la luz del sol se dividen en dos grupos: el primero corresponde a un enfoque de desempeño que establece algún requerimiento, tal como el número de horas de asoleamiento, cantidad de radiación solar, entre otros. El segundo es un enfoque descriptivo donde la geometría de los edificios es definida por las alturas reguladas de los edificios.

Utilizando estos dos enfoques, las regulaciones de la accesibilidad solar se pueden aplicar de tres formas distintas:

- El nivel básico, basado en un enfoque de desempeño, define la cantidad requerida de radiación solar por cada orientación, ubicación urbana y zona climática. Esto permite libertad en el diseño, por lo que un diseńador que use este método debe demostrar que cumple con los requerimientos.

- El segundo nivel, también basado en un enfoque de desempeño, indica la cantidad de horas de asoleamiento que cumplen con los requisitos de radiación solar. El diseñador tiene que presentar las pruebas de que los edificios circundantes se mantienen expuestos al sol durante ese tiempo.

- El tercer nivel es un método descriptivo/prescriptivo, basado en las horas de asoleamiento requeridas. El método presenta el uso de Líneas de Sección como una herramienta simple para el diseño de acceso solar. El diseño según estas Líneas de Sección asegura la exposición solar de los edificios circundantes y de los espacios abiertos, sin la necesidad de demostrar requisitos adicionales. 
A nivel internacional encontramos diferentes requerimientos de acceso solar. Por ejemplo, en Buenos Aires (Argentina) se establece que las edificaciones nuevas de tipología aislada deben emplazarse de tal manera que se aseguren dos horas de sol en el solsticio de invierno en al menos el $50 \%$ de los locales de primera clase o habitables de cada unidad de vivienda, mientras que cuando se edifique entre medianeras se permite que hasta el $50 \%$ de las unidades de viviendas no reciban asoleamiento directo, siempre que se asegure un ambiente interior de $18^{\circ} \mathrm{C}$ contra $0^{\circ} \mathrm{C}$ en el exterior y que exista ausencia de humedad por condensación. El restante $50 \%$ de unidades de vivienda debe cumplir con los requisitos para viviendas aisladas (Municipalidad de Buenos Aires, 2017). En el Reino Unido, el Building Research Establishment recomienda que al menos la mitad del área del jardín reciba como mínimo dos horas de sol el 21 de marzo (Littlefair, 2011). En otros países se han creado regulaciones desde el punto de vista público para mantener espacios abiertos (parques, pasajes, calles, etc.) y las veredas peatonales asoleadas; ejemplos de tales normativas son las definidas en ciudades como Toronto (Bosselman, Arens, Dunker \& Wright, 1995), y Tel Aviv (Capeluto, Yezioro \& Shaviv, 2003). En Chile, además de lo exigido en la oGUC, existen recomendaciones de asoleamiento complementarias para proyectos de viviendas sociales, que consisten en favorecer la orientación hacia el ecuador de al menos una cara de la vivienda. Esto para obtener el mayor porcentaje de asoleamiento en viviendas y así mejorar las condiciones de confort de sus usuarios (MINVU, 2017).

\section{Herramienta de diseño: Líneas de Sección}

Capeluto, Yezioro, Bleiberg y Shaviv (2005) describen una herramienta de diseño para el aseguramiento de acceso solar basada en el concepto de envolvente solar, denominada Líneas de Sección. Estas líneas representan el ángulo de la posición del sol sobre el horizonte más bajo para un espacio de tiempo requerido y están relacionadas con el concepto de Ángulo de Horizonte Urbano (UHA, por sus siglas en inglés) de un cañón urbano (Figura 1). El uHA es el ángulo subtendido desde una altura $h$, hasta la cima de los edificios contrarios $(\mathrm{H})$, separados por una calle de ancho W. La Línea de Sección corresponde al uHa que permite el acceso directo del sol hasta el punto P durante un periodo para un día determinado; por lo tanto, define la altura de los edificios contrarios para satisfacer tal restricción.

Los edificios que se ubiquen por debajo del plano que genera al ángulo propuesto por las Líneas de Sección no bloquearán el sol a las edificaciones vecinas al menos durante las horas y días requeridos. Capeluto et al. (2006) presentan las Líneas de Sección en una serie de nomogramas definidos para cuatro zonas térmicas de Israel, donde se muestra que las Líneas de Sección poseen distintos valores de ángulos críticos que permiten asegurar ciertas horas de exposición solar. Estos valores críticos dependen del momento del año (días y horas) en que se quiera asegurar el asoleamiento y de la orientación de la fachada sobre la que se quiera asegurar la visibilidad del sol. 
Figura I | Perfil de un cañón urbano y sus definiciones de H, w, uha, P y h

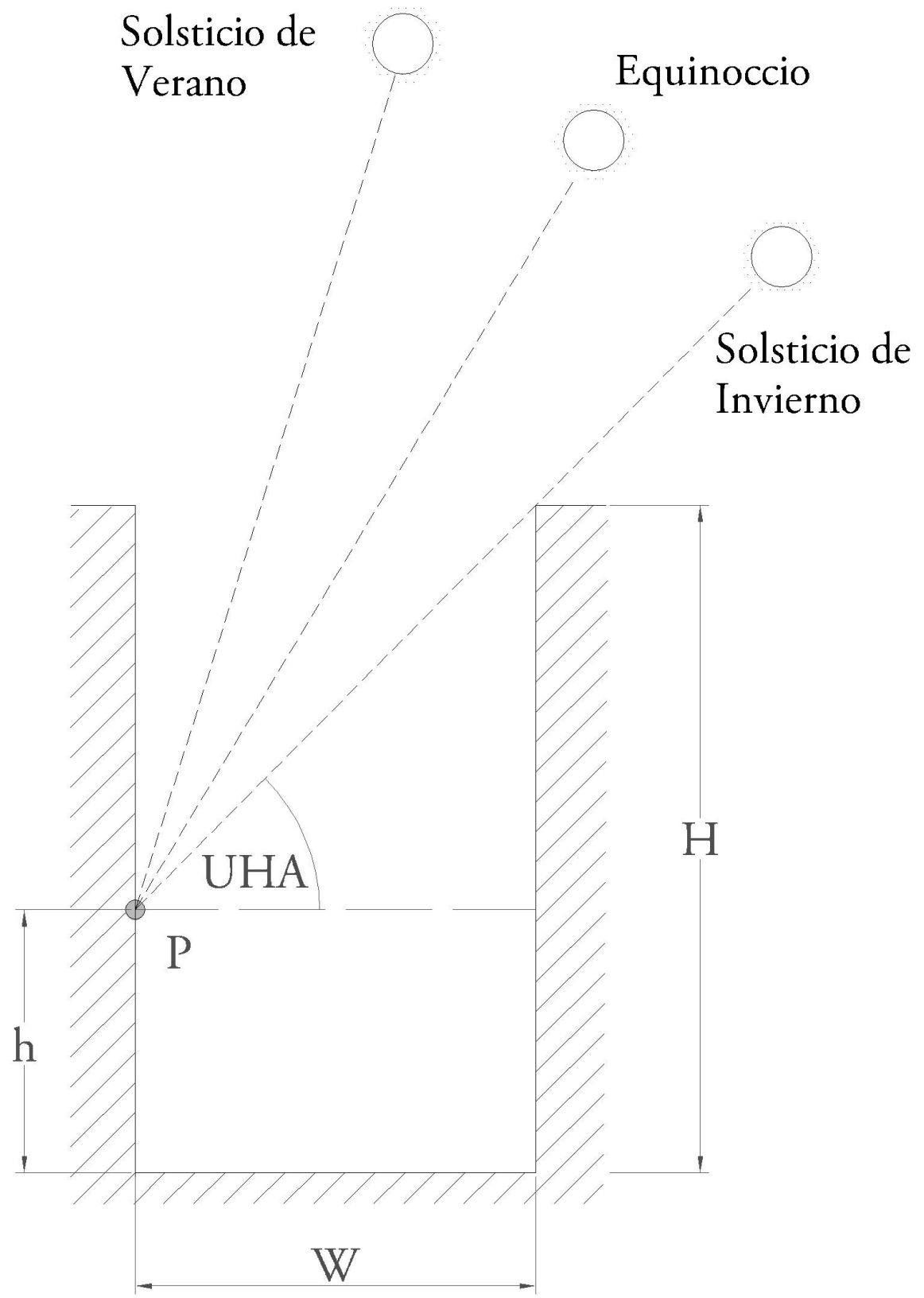

FUENTE: ELABORACIÓN PROPIA 
La Figura 2 muestra la aplicación de las Líneas de Sección que permiten asegurar el asoleamiento del punto medio del primer nivel (P) de un edificio emplazado en Tel Aviv (latitud geográfica aproximada de 32 grados norte), al limitar las alturas máximas de las edificaciones circundantes en dos orientaciones: la cara de un edificio orientada hacia el este (derecha) y la cara de otro edificio orientada hacia el norte (izquierda). Tal y como se esperaba, las obstrucciones de las edificaciones que estén orientadas hacia la línea del ecuador tendrán una baja altura para permitir su asoleamiento. Cabe destacar que el punto P de la Figura 2 puede ubicarse a una altura definida por el urbanista en función de la zona en la que se desee privilegiar el asoleamiento. Por ejemplo, en las zonas centrales de la ciudad, donde los primeros pisos suelen ser usados para fines comerciales, el punto $\mathrm{P}$ se puede definir en el segundo nivel. Esto permitiría construir edificios más altos.

FIgURA 2 Altura de los edificios en Tel Aviv para permitir el acceso solar. El primer piso es residencial y los edificios están separados por un ancho de calle W. a) Aseguramiento de acceso solar en las fachadas; y b) Aseguramiento de acceso solar en las aceras de ancho igual a $s$
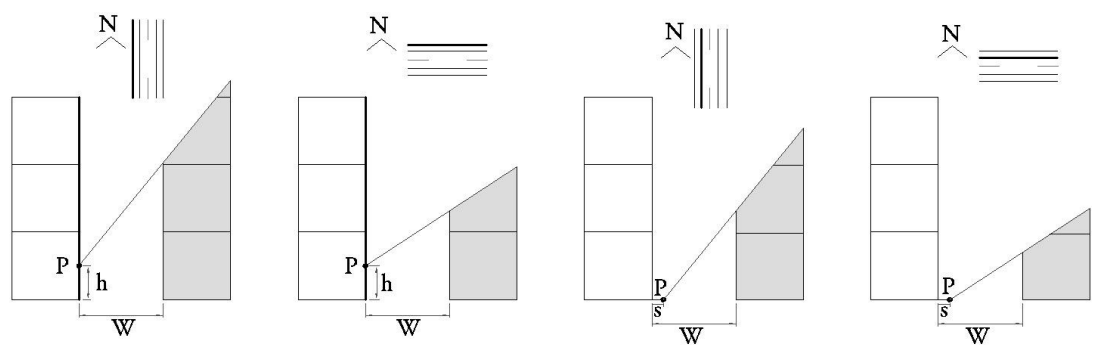

FUENTE: ELABORACIÓN PROPIA A PARTIR DE CAPELUTO ET AL. 2006

La aplicación de esta herramienta difiere según se requiera mantener el acceso solar para edificios, aceras peatonales o espacios públicos como plazas. Los requerimientos para asoleamiento de aceras son aquellos en que al menos uno o dos metros de su ancho estén asoleados, considerando una acera a la vez. En áreas del centro de la ciudad, en donde se requieren densidades mayores, es posible asegurar el asoleamiento de solo un metro de la acera. Los dos escenarios de la derecha (parte b) de la Figura 2 muestran la aplicación de las Líneas de Sección en el caso de aceras. Se puede observar que, en este caso, el punto P está emplazado a nivel del suelo a una distancia que puede ser uno o dos metros desde la cara del edificio, según la superficie en que se desee asegurar el asoleamiento. La aplicación de las Líneas de Sección en un espacio abierto es más compleja que para edificios y aceras. Esto debido a que los edificios rodean el espacio abierto desde todas las direcciones; por lo tanto, mantener las horas críticas de cada orientación no es suficiente, principalmente porque las orientaciones este y oeste permiten alturas de edificios más altas. En este trabajo, solo se presentarán las Líneas de Sección para edificios residenciales. 


\section{Metodología}

El objetivo de este artículo es presentar una herramienta para la incorporación del acceso solar en la planificación urbana a través del uso de las Líneas de Sección en el contexto chileno. Chile posee un desarrollo latitudinal extenso, que comprende de los $17^{\circ}$ a los $56^{\circ}$ de latitud sur. Por lo tanto, la geometría solar (posición y trayectoria del sol) cambia enormemente a lo largo del territorio chileno, lo que justifica tener una herramienta de incorporación del acceso solar a las edificaciones que sea sensible a la variación de la latitud geográfica.

Para abarcar la mayor parte del territorio nacional y analizar los efectos de la trayectoria solar, se seleccionaron siete ciudades chilenas situadas en distintas latitudes geográficas: Iquique $\left(20^{\circ} \mathrm{I} 3^{\prime}\right)$, Copiapó $\left(27^{\circ} 22^{\prime}\right)$, Coquimbo $\left(29^{\circ} 57^{\prime}\right)$, Concepción $\left(36^{\circ} 50^{\prime}\right)$, Temuco $\left(38^{\circ} 45^{\prime}\right)$, Osorno $\left(40^{\circ} 34^{\prime}\right)$ y Punta Arenas $\left(53^{\circ} 09^{\prime}\right)$. Estas siete ciudades corresponden a zonas urbanas altamente densificadas con una población superior a los 125.000 habitantes y que poseen zonas residenciales, zonas comerciales, avenidas, plazas, etc..

La Figura 3 muestra la variación del ángulo de elevación del sol respecto de la horizontal en el mediodía solar (posición más alta en el cielo) durante el año, para las ciudades en estudio. A medida que aumenta la latitud (hacia el sur), la altura solar disminuye, lo que implica que mientras más al sur se emplace una vivienda, más restrictivo debe ser el ángulo de aseguramiento solar.

\section{FIGURA 3 | Alturas solares al mediodía durante un año completo para las ciudades en estudio}

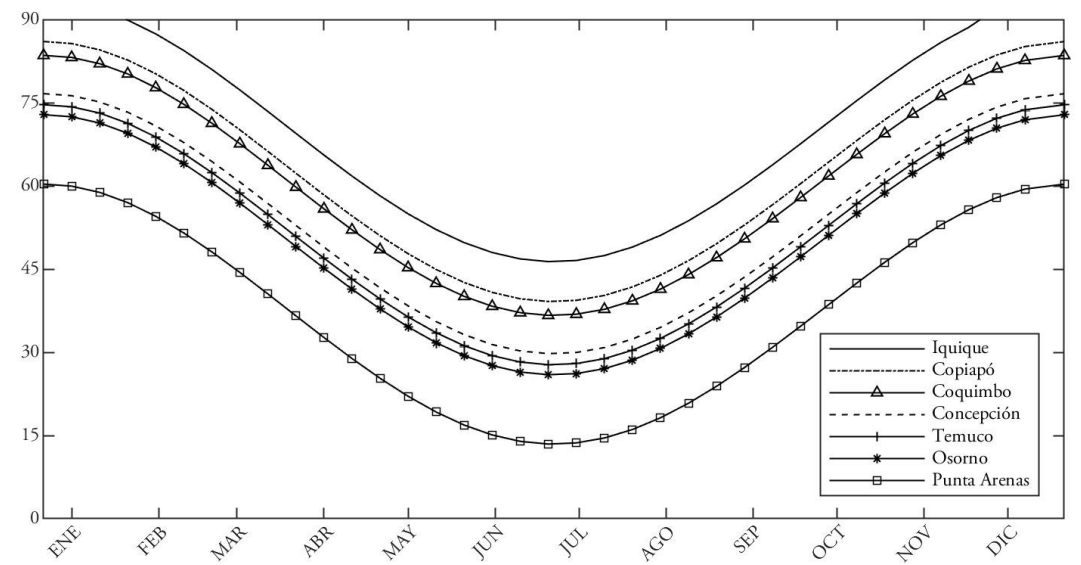

FUENTE: ELABORACIÓN PROPIA

\section{Periodo de evaluación}

El estándar utilizado para la creación de las Líneas de Sección corresponde al aseguramiento de una hora de acceso solar para el día más desfavorable del año, es decir, para el día en el cual la altura solar al mediodía es más baja. Este día corresponde al 
solsticio de invierno en el hemisferio sur (21 de junio), el cual se puede observar en la parte baja de las curvas de la Figura 3. Este estándar permite asegurar el acceso del sol a las edificaciones durante el año completo en sus fachadas norte, este y oeste.

El ángulo de la Línea de Sección de cada localidad dependerá del estándar escogido, es decir, cambiará según el requerimiento de horas de acceso solar. Por lo tanto, se hará un análisis de sensibilidad del ángulo obtenido en las Líneas de Sección al asegurar 30 minutos, una, dos y tres horas de exposición solar. Con esto, se podrá determinar el efecto producido en el volumen de la envolvente obtenida con las Líneas de Sección, de acuerdo con el requerimiento horario.

Aplicación de las Líneas de Sección: configuración urbana de manzanas y calles El plano de las ciudades tradicionales chilenas tiene la forma de un tablero de ajedrez, donde la ciudad se organiza mediante el diseño de sus calles en ángulo recto, creando manzanas rectangulares y ortogonales. Este tipo de diseńo urbano fue transmitido por los espańoles a sus colonias en América y se mantuvo entre los siglos XVI y xix (Garretón, 1997). Actualmente se encuentra a lo largo de todo el territorio nacional, incluyendo las ciudades en estudio.

Para mostrar la aplicación de las Líneas de Sección en el contexto urbano, se ocupa como grilla urbana una manzana ortogonal de 100 x 100 metros orientada hacia el norte geográfico, con una separación entre líneas oficiales de 15 metros (distancia entre las caras de los edificios opuestos), que corresponde a una vía de servicio unidireccional descrita en el Artículo 2.3.4 de la oguc (minvu, 2018). Se presentará la envolvente solar formada por las Líneas de Sección en Concepción; para observar el efecto de la grilla urbana en el volumen de la envolvente solar, se consideran tres tipologías de tejido urbano (Figura 4). Las tipologías 1 y 3 de la Figura 4 corresponden a una grilla de $100 \times 100$ metros que es atravesada en el sentido horizontal y vertical, respectivamente, por una vía local de 15 metros, que las separa en dos predios de $100 \times 42,5$ metros.

FIgURA 4 | Tipologías de grilla urbana sobre las que se aplicarán las Líneas de Sección

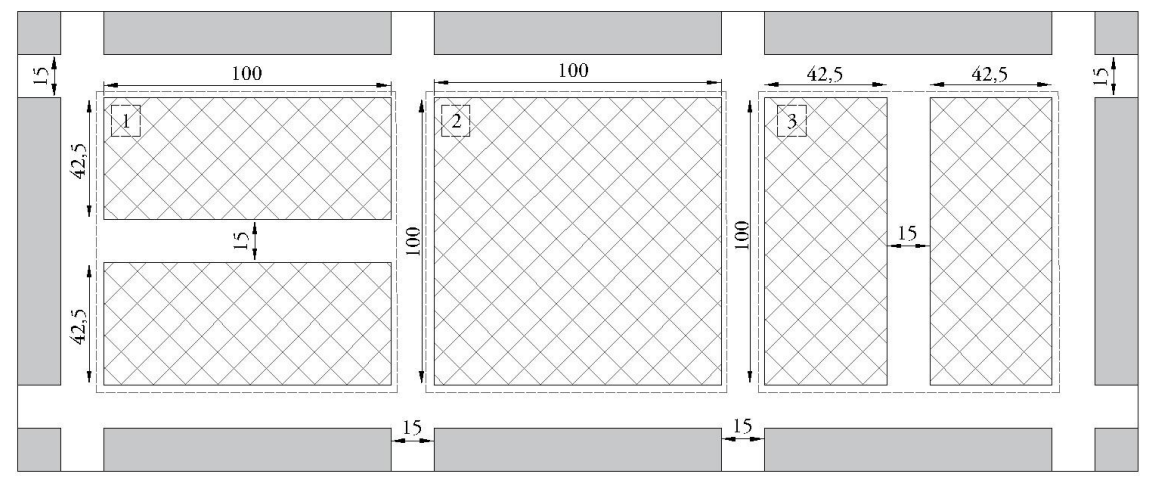

FUENTE: ELABORACIÓN PROPIA 


\section{Consideraciones de las Líneas de Sección}

En este artículo solo se tratarán las Líneas de Sección para aseguramiento solar en las fachadas de los edificios y no se analizará el caso de asoleamiento en aceras ni en el espacio público. Las Líneas presentadas no hacen consideración de las zonas climáticas ni la ubicación espacial de las ciudades analizadas; además, solo se presentarán para las orientaciones norte y este-oeste. El punto base desde el cual nacen las líneas es de 1,5 metros sobre el nivel del suelo, que representa la altura del punto medio de una ventana del primer nivel.

\section{Caso de estudio: simulación energética en Concepción}

Las simulaciones energéticas permiten evaluar el impacto de la aplicación de las Líneas de Sección en distintos escenarios de planificación urbana y realizar comparaciones cuantitativas entre distintas propuestas (Compagnon, 2004). Por este motivo, se hará una comparación de los aportes solares que inciden sobre los edificios que cumplen con el criterio de la rasante y con las Líneas de Sección. Para ello, se propone una representación geométrica de las ciudades como un tejido urbano periódico donde el elemento básico, llamado célula urbana (Vermeulen, Merino, Knopf-Lenoir, Villon \& Beckers, 2018), se repite en cada dirección, produciendo máscaras solares para sí misma.

En este caso, la célula urbana se construirá subdividiendo una manzana de $100 \times 100$ metros en nueve predios cuadrados e idénticos de $33 \times 33$ metros. Las edificaciones que se emplacen en cada predio dejarán un distanciamiento a cada deslinde de tres metros, lo que generará torres de tipología aislada y de base cuadrada de arista igual a 27 metros; es decir, un porcentaje de ocupación del suelo de cada predio igual a $67 \%$.

Se analizó la radiación solar global que incide sobre las fachadas de cada edificio. Para esto, se utilizó el código de simulación de energía solar uRBES, que puede manejar grandes escenarios urbanos (Merino, 2013; Vermeulen, 2014; Wegertseder, Lund, Mikkola \& García, 2016). Los datos de entrada principales del código URBES son de la morfología urbana sobre la cual se quiere determinar la radiación solar incidente y la latitud geográfica del lugar donde está emplazado el proyecto, ya que esta define el trayecto del sol durante el año. Para las simulaciones, se usó un modelo de radiación solar para días despejados (Liu \& Jordan, 1960), lo que permite una ponderación real de las posiciones del sol a lo largo de un día específico, de modo que la radiación solar recibida al amanecer o atardecer tiene una menor influencia que la radiación recibida al mediodía (Vermeulen et al., 2018).

La simulación fue realizada para los 30 días más desfavorables del año, es decir, cuando las alturas solares son mínimas, y corresponden al periodo situado entre el 6 de junio y el 6 agosto (entre los días 157 y 187 del calendario), utilizando un paso de tiempo de una hora. La resolución espacial del mallado urbano corresponde a una malla rectangular de $5 \times 5$ metros, aproximadamente. La principal salida es la radiación solar global incidente en cada superficie (suma de la radiación solar directa, difusa y reflejada), renderizada en un mapa del área urbana que permite identificar las áreas afectadas por el sombreamiento de edificios vecinos (Merino et al., 2015). 


\section{Resultados}

En esta sección se presentan las Líneas de Sección para cada una de las ciudades en estudio. Además, se muestra un ejemplo de aplicación, utilizando como caso de estudio la ciudad de Concepción. Se presentan las envolventes solares formadas por las Líneas de Sección en distintos patrones de tejido urbano. Finalmente, se comparan las envolventes solares con las envolventes de la rasante a través de una simulación energética de la radiación solar total incidente en fachadas.

\section{Líneas de Sección}

La Figura 5 muestra las Líneas de Sección que permiten asegurar una hora de acceso solar durante el solsticio de invierno en las orientaciones norte, este y oeste de las viviendas ubicadas en siete ciudades chilenas.

FIGURA 5 | Líneas de Sección para siete ciudades chilenas. Las Líneas de Sección presentadas permiten asegurar 1 hora de acceso solar para el solsticio de invierno
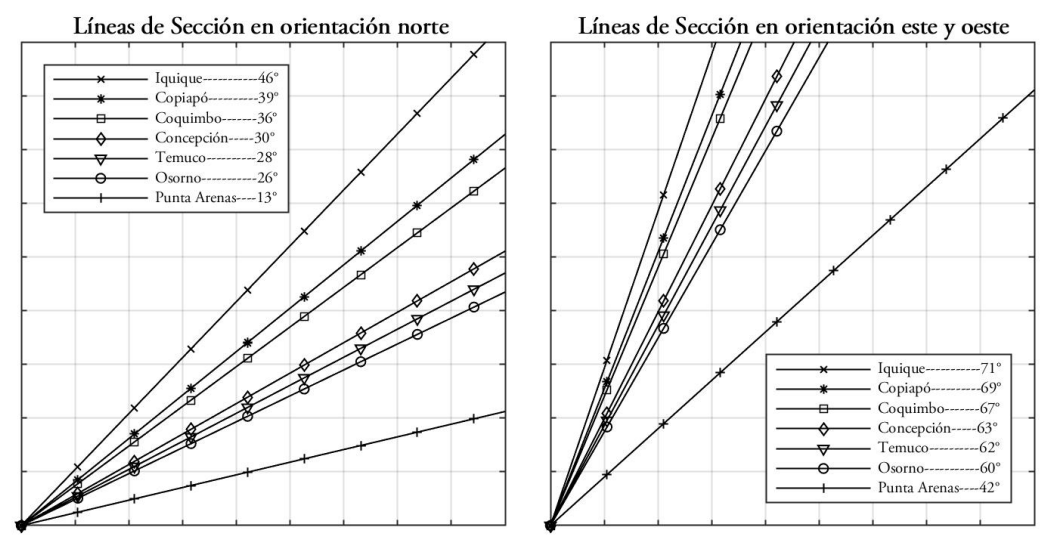

FUENTE: ELABORACIÓN PROPIA

En este caso, para determinar la altura máxima de la fachada opuesta a una orientación dada (H) de las edificaciones, se debe multiplicar el ancho entre líneas oficiales $(\mathrm{W}=15 \mathrm{~m})$ por la tangente del ángulo $(\alpha)$ definido por la Línea de Sección, y sumar este resultado a la altura del punto $\mathrm{P}$ sobre el nivel del suelo, que en este caso se definió como 1,5 metros, es decir $\mathrm{H}=1,5+\tan (\alpha)$. Por lo tanto, al aplicar las Líneas de Sección de la Figura 5 para determinar la altura máxima de la fachada opuesta de un edificio con orientación norte en cada una de las ciudades incluidas en este estudio, se obtiene que en Iquique se puede edificar aproximadamente hasta los 17 metros de altura; en Copiapó, 13 metros; Coquimbo, 12 metros; Concepción, 10 metros; Temuco, 9 metros; Osorno, 8 metros; y Punta Arenas, 5 metros. Sin embargo, al aplicar las Líneas de Sección para determinar la altura de las fachadas orientadas hacia el este y oeste, se obtiene que en Iquique se puede 
edificar aproximadamente hasta los 45 metros de altura; en Copiapó, 40 metros; Coquimbo, 37 metros; Concepción, 31 metros; Temuco, 29 metros; Osorno, 27 metros; y Punta Arenas, 15 metros. Estas diferencias de altura para las fachadas norte, este y oeste en una misma ciudad generan los volúmenes máximos de edificación con forma de pirámide que se muestran en la Figura 6.

El ángulo definido por la Línea de Sección genera un plano imaginario que limita la altura de edificación con el objetivo de asegurar niveles mínimos de asoleamiento. Esto es similar a la rasante definida en la oguc, pero con la diferencia de que la superficie imaginaria inclinada nace desde el nivel del suelo hacia el interior de la propiedad, para limitar la altura de edificación del proyecto que se emplace en ella. Por el contrario, en el caso de la Línea de Sección, la superficie imaginaria puede nacer desde el nivel del suelo u otra altura definida por el proyectista hacia el exterior de la propiedad, con el objetivo de limitar la altura de las edificaciones vecinas. Esta diferencia conceptual entre la rasante y la Línea de Sección puede generar confusiones al momento de comparar los ángulos definidos en estas dos herramientas de planificación. Por ejemplo, en la ciudad de Concepción la rasante es de 60 grados sexagesimales, y la Línea de Sección tiene dos valores: 30 grados para una fachada orientada hacia el norte y 63 grados para fachadas este y oeste. Estas diferencias generan una altura máxima de 10 metros para una edificación frente a una fachada orientada hacia el norte al usar la Línea de Sección, pero al usar la rasante, la altura aumenta a 20 metros. Sin embargo, como la rasante no discrimina por orientación, genera exactamente la misma altura para todas las fachadas; es decir, 20 metros. Al usar la Línea de Sección, se determina que la altura máxima de una edificación orientada hacia el norte aumenta sustancialmente respecto de la rasante y llega a los 66 metros, como se muestra en la Figura 6. Acá se han presentado las alturas máximas de las fachadas, pero cabe destacar que, en el caso de las edificaciones emplazadas frente a aquellas orientadas hacia el norte, la altura es de 10 metros, pero se puede construir superando este límite si la edificación se emplaza hacia el interior de la propiedad, dejando un antejardín o similar.

Las alturas máximas de edificación de las construcciones que se emplacen frente a la fachada orientada hacia el norte, determinadas por las Líneas de Sección, son de aproximadamente 17 metros en Iquique, 13 metros en Copiapó, 12 metros en Coquimbo, 10 metros en Concepción, 9 metros en Temuco, 8 metros en Osorno y 5 metros en Punta Arenas. La altura es diferente en cada ciudad y decrece de norte a sur debido a que los rayos solares presentan una inclinación más baja en las latitudes geográficas alejadas de la línea del ecuador. Sin embargo, al usar el criterio de la rasante que divide al país en tres zonas, obtenemos que en Iquique y Copiapó la altura máxima es la misma, e igual a 42 metros; en Coquimbo, Concepción y Temuco la altura también es la misma, e igual a 20 metros. Finalmente, en Osorno y Punta Arenas la altura máxima es de 13 metros.

\section{Aplicación de las Líneas de Sección en Concepción}

La Figura 6 muestra la envolvente solar que se obtiene al aplicar las Líneas de Sección de la Figura 5 para la ciudad de Concepción, en las tipologías de grillas de la Figura 4. Cualquier edificio que se encuentre por debajo de esta envolvente 
permitirá el acceso del sol a las edificaciones cercanas bajo el requerimiento horario especificado en la sección anterior.

FIGURA 6 | Envolventes solares generadas en grillas urbanas de $100 \times 100$ metros aplicando las Líneas de Sección. a) Vista en perspectiva; b) Vista frontal y c) Vista lateral

a)

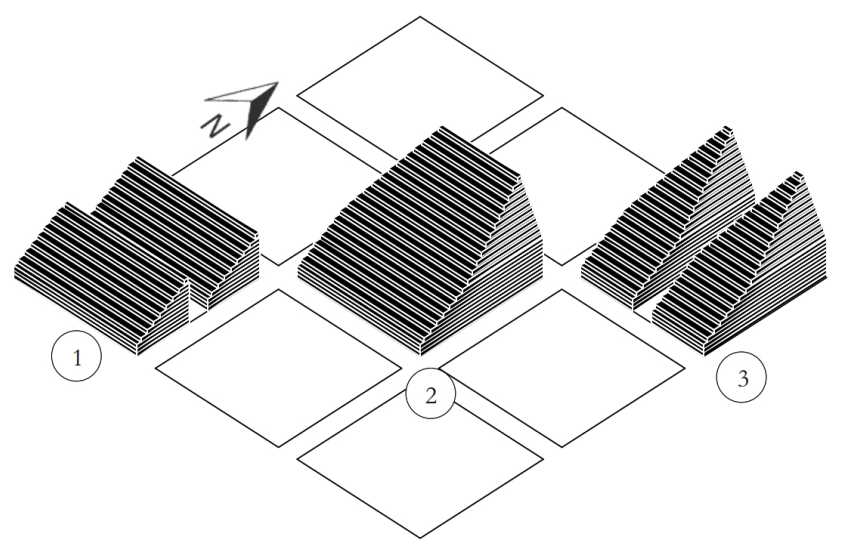

b)
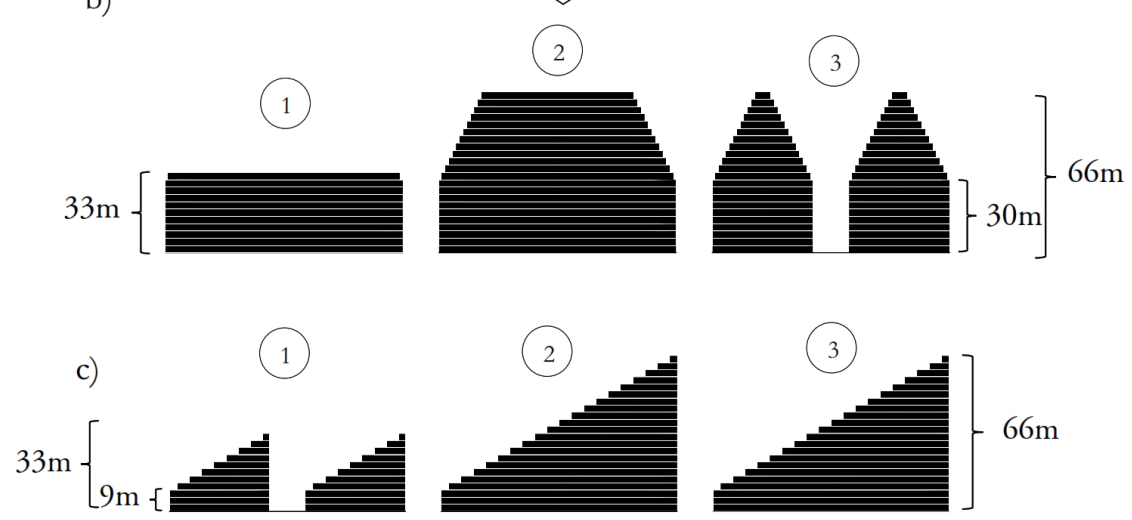

FUENTE: ELABORACIÓN PROPIA

Las Líneas de Sección generan envolventes con geometrías escalonadas, que alcanzan una altura máxima de 66 metros en la zona norte de la grilla urbana. La forma de la grilla tiene un fuerte impacto en el volumen de la envolvente solar, ya que una grilla orientada de norte a sur (Envolvente 3 de la Figura 6) permite un volumen 39\% mayor que la grilla orientada de este a oeste (Envolvente 1 de la Figura 6). Las tres tipologías mostradas en la Figura 6 presentan diferentes volúmenes de edificación, donde la tipología tipo 1 tiene un volumen total de 177.872 metros cúbicos. La tipología tipo 3 tiene un volumen 39\% mayor a la tipología 1 y la tipología tipo 2 
tiene un volumen $4 \%$ mayor a la tipología tipo 1 . Por otro lado, al comparar el número de pisos de estas tipologías, encontramos que la tipología tipo 1 tiene 11 pisos, y que las tipologías tipo 2 y 3 tienen el mismo número de pisos, igual a 22 .

Análisis de sensibilidad: horas de asoleamiento

El ángulo de las Líneas de Sección de la Figura 5 está definido para permitir una hora de acceso solar; por lo tanto, será distinto para cualquier otro requerimiento. En la Figura 7 se presentan las Líneas de Sección para Concepción que aseguran 30 minutos, 1 hora, 2 horas y 3 horas de acceso solar.

FIGURA 7 | Líneas de Sección para aseguramiento de 30 minutos, 1 hora, 2 horas y 3 horas durante el solsticio de invierno para la ciudad de Concepción
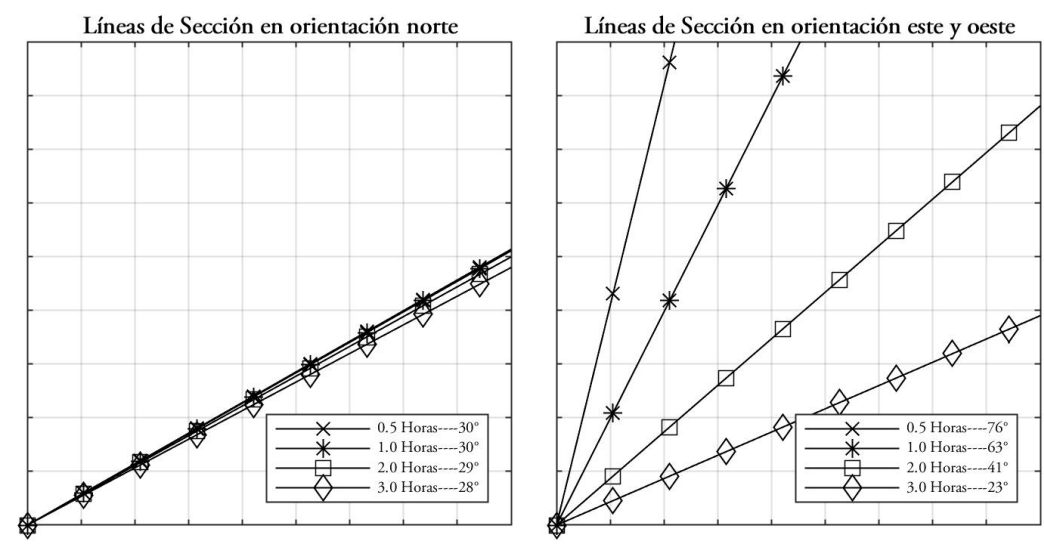

FUENTE: ELABORACIÓN PROPIA

En los nomogramas de la Figura 7, para la orientación norte los ángulos de las Líneas de Sección no cambian de manera significativa al variar las horas de acceso solar. Sin embargo, para las orientaciones este y oeste, se observa una disminución importante en el ángulo al aumentar la cantidad de horas requeridas, pasando desde los 76 grados para 30 minutos de asoleamiento hasta 23 grados para 3 horas. Esta gran variación del ángulo de la Línea de Sección muestra nuevamente la sensibilidad de esta herramienta a la orientación de las obstrucciones del sol, a diferencia de la rasante.

Para ilustrar más claramente cómo la incorporación del número de horas de asoleamiento influye en el volumen edificable, se utilizó la célula solar definida en la sección de Metodología. El volumen máximo de edificación obtenido con las Líneas de Sección del nomograma de la Figura 5, que asegura una hora de asoleamiento en el solsticio de invierno, entregó 380.000 metros cúbicos. Al disminuir la exigencia a media hora de asoleamiento, el volumen aumenta solo un $4 \%$. Sin embargo, al aumentar la exigencia y asegurar dos horas de asoleamiento, se produce una disminución en un $28 \%$ en el volumen de la envolvente solar, al compararlo 
con el volumen para aseguramiento de una hora, mientras que para tres horas la disminución es aún mayor, llegando al 56\%. Lo anterior se traduce en una disminución de las alturas de edificación. La siguiente sección explora más en detalle la morfología de la célula urbana para el aseguramiento del asoleamiento de una hora en el solsticio de invierno.

\section{Simulación energética}

La Figura 8 muestra la morfología de nueve edificaciones obtenida al usar el criterio de aseguramiento solar de la rasante estipulada en la ogUC (izquierda) y el propuesto de Líneas de Sección (derecha). Los edificios están emplazados en la ciudad de Concepción sobre predios cuadrados e idénticos de 33 ×33 metros. Para incorporar las obstrucciones del sol de las edificaciones circundantes, se incluyeron muros en las orientaciones norte, este y oeste, cuyas alturas respetan los criterios de aseguramiento solar. La Figura 8 muestra cómo el criterio de la rasante (izquierda) no discrimina por orientación, generando una morfología de tipo piramidal escalonada. Por el contrario, el criterio de Líneas de Sección sí incorpora la orientación al limitar la altura de las edificaciones vecinas, lo que genera una forma escalonada cuya superficie orientada hacia la línea del ecuador es la más grande, lo que maximiza la captación solar en periodos de invierno y minimiza el consumo de energía para calefaccionar las edificaciones. Cabe destacar que, para evitar el sobrecalentamiento de las edificaciones en periodos estivales, el diseño debe incorporar protecciones solares que bloqueen los rayos solares con un ángulo de incidencia mayor que los rayos invernales.

FIGURA 8 | Morfología de nueve edificios vecinos obtenidos al usar el criterio de aseguramiento de asoleamiento de a) rasante y b) Líneas de Sección

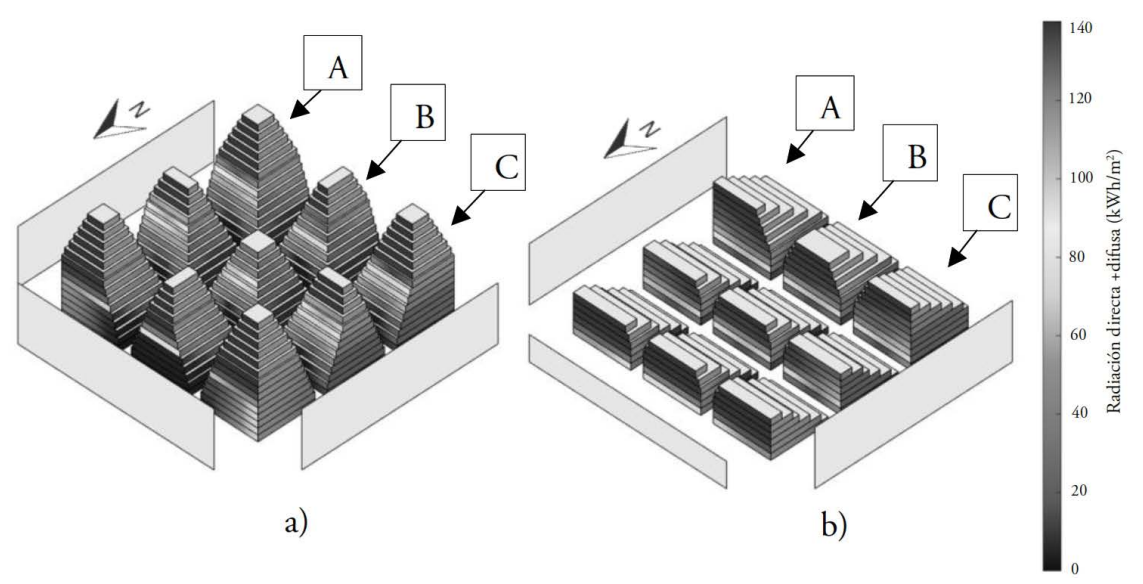

FUENTE: ELABORACIÓN PROPIA 
Además, la Figura 8 muestra que la altura de los edificios creados utilizando la rasante es mayor que la de los edificios creados utilizando las Líneas de Sección. Sin embargo, esto tiene un impacto negativo en la cantidad de energía solar recibida sobre las fachadas de los edificios. Por ejemplo, en la fachada norte de los edificios A, B y C se encuentran diferencias considerables en cuanto a la radiación global incidente, principalmente en los tres primeros niveles, tal como se observa en la Figura 9.

\section{FIGURA 9 | Comparación de la radiación incidente sobre fachada norte para las torres $\mathrm{A}, \mathrm{B}$ y $\mathrm{C}$, en los tres primeros pisos de altura}

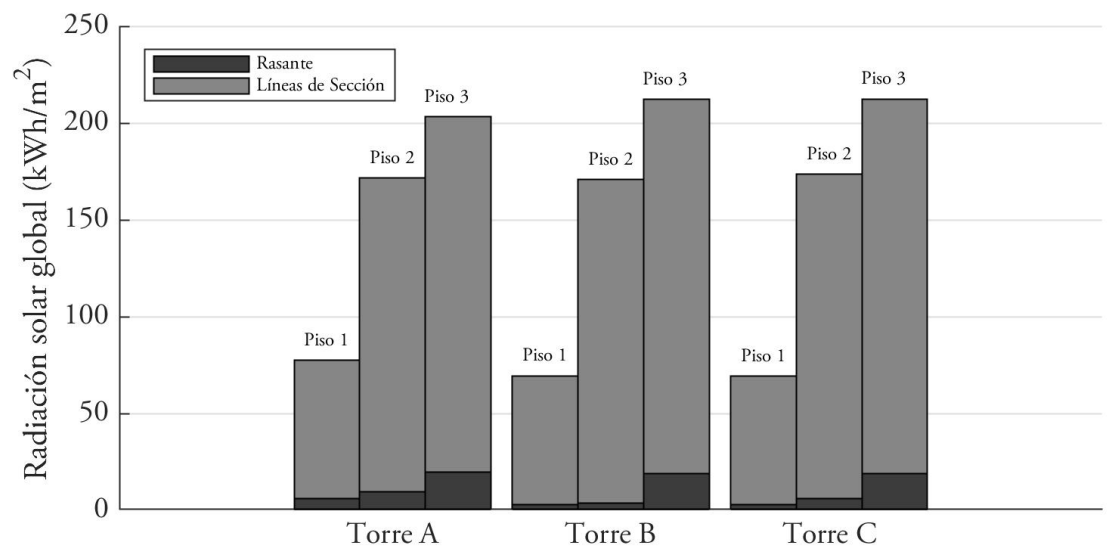

FUENTE: ELABORACIÓN PROPIA

En la Figura 9 se observa que, en el periodo de simulación, existen diferencias importantes en la exposición solar en la fachada norte entre los edificios que cumplen con la rasante y los creados siguiendo el criterio de las Líneas de Sección. Estas diferencias se observan principalmente en los primeros pisos. En promedio, el primer piso de los edificios creados con la rasante recibe un 5,83\% del aporte solar que reciben los edificios creados con las Líneas de Sección; el segundo piso recibe en promedio el 3,96\% y el tercero, un 10\%. Por otro lado, aquellos edificios creados aplicando el criterio de la rasante generan grandes zonas de sombreamiento sobre las edificaciones cercanas, en las cuales se ve disminuido el acceso solar de los primeros pisos. Una disminución en el acceso solar se traduce en un menor aporte energético en los meses de invierno, lo que incrementa la demanda de calefacción e iluminación de las torres afectadas.

\section{Conclusiones}

En este trabajo se presentó una herramienta útil para orientar de manera sencilla las normas urbanísticas que condicionan la morfología edilicia con el fin de asegurar el acceso solar en edificaciones de un medio urbano. Esta herramienta se denomina 
Líneas de Sección y entrega el ángulo de elevación máximo de una superficie imaginaria, la cual no debe ser sobrepasada, ya que cualquier edificio con una altura mayor producirá obstrucciones del sol a las edificaciones vecinas. Esta herramienta es especialmente útil para los planificadores en la creación de diseños edilicios más sustentables.

En Chile, las zonas definidas con el criterio de la rasante abarcan un rango latitudinal muy amplio; por lo tanto, dicho criterio no se adapta a las características de la trayectoria solar de cada región del país, tales como la altura solar, cantidad de radiación, etc.. Un diseño que respete la norma de la rasante se puede mantener igual en dos ciudades de latitudes distintas, pero ellas tendrán diferentes disposiciones al acceso solar, a la cantidad de radiación e iluminación que reciben. Por el contrario, las Líneas de Sección se acomodan de acuerdo con la latitud, lo que significa que se pueden adaptar según sean las características de la trayectoria solar de cada localidad chilena. A través de la herramienta propuesta en este artículo, se puede determinar que el criterio de la rasante puede ser mejorado al reducir sus ángulos de inclinación y aumentar la zonificación del territorio nacional.

Si se lo compara con el criterio de la rasante, el uso de las Líneas de Sección como criterio de acceso solar permite un mayor asoleamiento de las fachadas de los edificios, principalmente en los primeros pisos. La simulación de la energía solar recibida sobre la envolvente urbana en Concepción para un periodo de 30 días (desde el 6 de junio al 6 de agosto), evidencia que, al aplicar las Líneas de Sección, se puede captar 10 veces más energía solar en la fachada norte de los primeros pisos, en comparación con la rasante. Si bien aplicar las Líneas de Sección es ventajoso para una condición de invierno cuando se busca maximizar el aporte solar a las viviendas, en verano ello puede genera graves problemas de sobrecalentamiento al interior de las viviendas, lo que se soluciona fácilmente con el uso de protectores solares que eviten el sobrecalentamiento.

La implicancia urbana que tiene el aplicar la metodología de las Líneas de Sección y no la rasante, es que la primera restringe en mayor medida las alturas máximas de edificación. Esto significa que se condicionan las alturas máximas de edificación en función de una garantía mínima de acceso solar, compensándolo con altas condiciones de confort y oportunidades de aprovechamiento solar pasivo y activo. Adicionalmente, permite orientar la decisión sobre situaciones de agrupamientos de edificios (aislado, continuo o pareado en relación con el cañón urbano). Por esto, la aplicación de las Líneas de Sección se hace especialmente conveniente para su uso en el sector residencial y no en zonas de alta densificación. Por otro lado, otra implicancia muy importante es la morfología de la envolvente solar o volumen máximo edificable que genera la Línea de Sección con respecto a la rasante, ya que esta última no discrimina por orientación, lo que genera un volumen en forma de pirámide regular. Sin embargo, la Línea de Sección sí discrimina por orientación, lo que da curso a un volumen completamente diferente, privilegiando alturas máximas de edificación en las fachadas orientadas hacia el ecuador.

El uso de las Líneas de Sección permite también evaluar la combinación de las distintas normas urbanísticas relacionadas con la morfología urbana, de modo de buscar un mejor equilibrio entre los elementos de diseño, uno que garantice acceso 
solar suficiente para una buena habitabilidad y densidades urbanas sostenibles, que incluso pueden llegar a ser altas, dependiendo de la combinación de factores de diseńo y su agrupamiento. Esto se puede conseguir modificando el requerimiento de horas de exposición solar y el periodo del año requerido, lo cual puede ser tratado en trabajos futuros.

Las Líneas de Sección presentadas en este trabajo responden al requerimiento de cantidad de horas de acceso solar. Sin embargo, también pueden crearse de acuerdo con requerimientos de cantidad de radiación solar, o una combinación entre horas y cantidad de radiación, lo cual será estudiado en trabajos futuros.

\section{Referencias bibliográficas}

Baker, N. \& Steemers, K. (2000). Energy and environment in architecture: A technical design guide. Londres: Taylor \& Francis.

Bosselmann, E., Arens, E., Dunker, K. \& Wright, R. (1995). Urban form and climate: Case study, Toronto. Journal of the American Planning Association, 61(2), 226-239. https:// doi.org/10.1080/01944369508975635

Capeluto, I. G. \& Ochoa, C. (2017). Intelligent envelopes for high-performance buildings: Design and strategy. Cham, Suiza: Springer.

Capeluto, I. G. \& Shaviv, E. (1999). Modeling the design of urban fabric with solar rights considerations. IBPSA Conference Proceedings (pp. 1341-1347). Kioto, Japón. https:// pdfs.semanticscholar.org/b278/d1ad5e5784d5ffd0695e53cd92564cf6c763.pdf

Capeluto, I. G. \& Shaviv, E. (2001). On the use of 'solar volume' for determining the urban fabric. Solar Energy, 70(3), 275-280. https://doi.org/10.1016/S0038-092X(00)00088-8

Capeluto, I., Yezioro, A., Bleiberg, T. \& Shaviv, E. (2005). From computer models to simple design tools: Solar rights in the design of urban streets. Ninth International IBPSA Conference (pp. 131-138). Montreal, Canadá, agosto 15-18. https://pdfs. semanticscholar.org/386e/ac6e4e8c99262f4bd4cb4efe9feae3980a6c.pdf

Capeluto, I., Yezioro, A., Bleiberg, T. \& Shaviv, E. (2006). Solar rights in the design of urban spaces. Proceedings of PLEA 2006 Ginebra - The 23rd Conference on Passive and Low Energy Architecture. Ginebra, Suiza, septiembre 6-8. http://citeseerx.ist.psu.edu/ viewdoc/download?doi=10.1.1.548.3742\&rep=rep $1 \&$ type=pdf

Capeluto, I., Yezioro, A. \& Shaviv, E. (2003). Climatic aspects in urban design - A case study. Building and Environment journal, 38(6), 827-835. https://doi.org/10.1016/S03601323(02)00063-X

Compagnon, R. (2004). Solar and daylight availability in the urban fabric. Energy and Buildings, 36(4), 321-328. https://doi.org/10.1016/j.enbuild.2004.01.009

Daher, A. (2013). El sector inmobiliario y las crisis económicas. EURE, 39(118), 47-76. http:// dx.doi.org/10.4067/S0250-71612013000300003.

Garretón, J. (1997). El urbanismo en Chile: Conquista y Colonia. Concepción: Ediciones Universidad de Concepción. 
Hachem, C., Athienitis, A. \& Fazio, P. (2014). Energy performance enhancement in multistory residential buildings. Applied Energy, 116, 9-19. https://doi.org/10.1016/j. apenergy.2013.11.018

Instituto Nacional de Estadísticas (INE), Chile. (2018). Sintesis de resultados. Censo 2017. Santiago, Chile: INE. https://www.censo2017.cl/descargas/home/sintesis-deresultados-censo2017.pdf

Knowles, R. (1981). Sun rhythm form. Cambridge, MA: MIT Press.

Knowles, R. (2003). The solar envelope: Its meaning for urban growth and form. Energy and Buildings, 35(1), 15-25. https://doi.org/10.1016/S0378-7788(02)00076-2

Lechner, N. (2015). Heating, cooling, lighting: sustainable design methods for architects. Hoboken, NJ: John Wiley \& Sons.

Littlefair, P. (2011). Site layout planning for daylight and sunlight: A guide to good practice. Bracknell, Berkshire: IHS BRE Press.

Liu, B. \& Jordan, R. (1960). The interrelationship and characteristic distribution of direct, diffuse and total solar radiation. Solar Energy, 4(3), 1-19. https://doi.org/10.1016/0038$092 X(60) 90062-1$

McCann, C. (2008). A comprehensive review of solar access law in the United States: Suggested standars for model statue and ordinance. Solar America Board for Codes and Standards Report. Orlando, FL: Solar Energy Center, University of Central Florida. http://www. solarabcs.org/about/publications/reports/solar-access/pdfs/Solaraccess-full.pdf

Merino, L. (2013). Modélisation du rayonnement solaire pour la simulation thermique en milieu urbain. Tesis doctoral. Université de Technologie de Compiègne, Compiègne, Francia. http://www.theses.fr/2013COMP2115

Merino, L., Vermeulen, T., Medina, R., Nahön, R. \& Agurto, L. (2015). Indicadores de sustentabilidad basados en el metabolismo urbano: Un caso de estudio usando la morfología del medio urbano construido en la ciudad de Concepción. En R. Barra \& J. Rojas (Eds.), Desarrollo sustentable: Miradas interdisciplinarias de experiencias en Chile y Brasil (pp. 93-105). Concepción, Chile: Editorial vrim, Universidad de Concepción. https://www.academia.edu/38653641/Desarrollo_Sustentable_ Miradas_interdisciplinarias_de_experiencias_en_Chile_y_Brasil

Ministerio de Energía, Chile. (2015). Energía 2050, Política energética de Chile. Santiago, Chile: Ministerio de Energía. http://www.energia.gob.cl/sites/default/files/energia_2050_-_ politica_energetica_de_chile.pdf

Ministerio de Vivienda y Urbanismo (MINVu), Chile. (2017). Itemizado técnico de construcción para proyectos del Fondo Solidario de Elección de Vivienda. Santiago, Chile: MINvu. https://www.minvu.cl/wp-content/uploads/2019/05/Res_7713-16062017_Itemi zado-Tecnico.pdf

Ministerio de Vivienda y Urbanismo (MINvu), Chile. (2018a). Estándares de construcción sustentable para viviendas de Chile. Santiago, Chile: MINvu. https://csustentable. minvu.cl/estandares-cs/

Ministerio de Vivienda y Urbanismo (minvu), Chile. (2018b). Ordenanza General de Urbanismo y Construcción. (2016). Santiago, Chile: MINvu. https://www.minvu.cl/wpcontent/uploads/2019/05/OGUC-Febrero-2018-disposiciones-transitorias-vigenciainmediata_22893911895876224.pdf 
Municipalidad de Buenos Aires. (2017). Código de Planeamiento Urbano de la Ciudad de Buenos Aires. Buenos Aires, Argentina. https://www.buenosaires.gob.ar/sites/gcaba/files/eval_ asoleamiento_pasajes.pdf

Oke, T. R. (1987). Boundary layer climates. Londres: Methuen.

Okeil, A. (2010). A holistic approach to energy efficient building forms. Energy and Buildings, 42(9), 1437-1444. https://doi.org/10.1016/j.enbuild.2010.03.013

Organización de las Naciones Unidas (onu). (2018). World Urbanization Prospects: The 2018 Revision, Key Facts. https://population.un.org/wup/Publications/Files/WUP2018KeyFacts.pdf.

Ratti, C., Baker, N. \& Steemers, K. (2005). Energy consumption and urban texture. Energy and Buildings, 37(7), 762-776. https://doi.org/10.1016/j.enbuild.2004.10.010

Sadineni, S., Madala, S. \& Boehm, R. (2011). Passive building energy savings: A review of building envelope components. Renewable and Sustainable Energy Reviews, 15(8), 3617-3631. https://doi.org/10.1016/j.rser.2011.07.014

Saldías, M. (2016). Acceso solar en áreas urbanas de Chile y su impacto en el balance energético residencial. Memoria de título. Universidad de Concepción, Concepción, Chile.

Sadineni, S., Madala, S. \& Boehm, R. (2011). Passive building energy savings: A review of building envelope components. Renewable and Sustainable Energy Reviews, 15(8), 3617-3631. https://doi.org/10.1016/j.rser.2011.07.014

Vermeulen, T. (2014). Optimisation de formes urbaines soumises au rayonnement solaire. Tesis doctoral. Université de Technologie de Compiègne, Compiègne, Francia. https://tel. archives-ouvertes.fr/tel-01095637/document

Vermeulen, T., Merino, L., Knopf-Lenoir, C., Villon, P. \& Beckers, B. (2018). Periodic urban models for optimization of passive solar irradiation. Solar Energy, 162, 67-77. https:// doi.org/10.1016/j.solener.2018.01.014

Wegertseder, P., Lund, P., Mikkola, J. \& García, R. (2016). Combining solar resource mapping and energy system integration methods for realistic valuation of urban solar energy potential. Solar Energy, 135, 325-336. https://doi.org/10.1016/j.solener.2016.05.061 